\title{
INDICIAL EQUIVALENTS OF MULTIPARAMETER DEFINITENESS CONDITIONS IN FINITE DIMENSIONS
}

\author{
by PAUL BINDING* \\ (Received 17th February 1984)
}

\section{Introduction}

Let $T_{m}, V_{m n}$ be Hermitean linear operators on complex Hilbert spaces $H_{m}, m=1 \ldots k$. A nonzero column vector $\lambda=\left[\lambda_{0} \ldots \lambda_{k}\right]^{T} \in \mathbb{B}^{k+1}$ satisfying

$$
W_{m}(\lambda) x_{m}=0 \neq x_{m} \in H_{m}
$$

where

$$
W_{m}(\lambda)=\lambda_{0} T_{m}+\lambda_{1} V_{m 1}+\ldots+\lambda_{k} V_{m k}
$$

will be called an eigenvalue. This type of problem has been studied extensively by Atkinson [2] from the viewpoint of determinantal operators on the tensor product $H^{\otimes}=\otimes_{m=1}^{k} H_{m}$. We shall connect his work with more recent investigations $[5,7]$ of eigenvalue indices based on minimax principles for $W(\lambda)=\left(W_{1}(\lambda), \ldots, W_{k}(\lambda)\right)$, which can be viewed as an operator on $H^{\times}=\mathrm{X}_{m=1}^{k} H_{m}$.

We should perhaps point out that the distinction between the $T_{m}$ and the $V_{m n}$ reflects the relative simplicity of the $V_{m n}$ in certain differential equation problems, with corresponding implications for finite dimensional (e.g. difference equation) approximations. Indeed Atkinson's results in $H^{\otimes}$ have their roots in multiple Fourier expansion theorems for linked Sturm-Liouville problems, going back to Hilbert. Likewise the indicial results in $H^{\times}$are developments of corresponding oscillation theorems dating back to Klein and Bôcher. Incidentally, an oscillation theory for linked difference equations can be found in [1; Chapter 6].

From now on, we shall assume that

$$
d_{m}=\operatorname{dim} H_{m}
$$

is finite. We shall discuss (1.1) under certain "definiteness conditions" which have received much recent attention. Our aim will be to relate the $H^{\otimes}$ and $H^{\times}$theories, together with certain geometrical equivalents of these definiteness conditions [2; Chapter 9], [3]. In particular, we shall demonstrate equivalence of some of the definiteness conditions with certain indicial properties of the eigenvalues $\lambda$ of (1.1).

The simplest case is that of right definiteness (RD) which requires

$$
0 \neq \delta_{0}(x):=\operatorname{det}\left[v_{m n}(x)\right]_{1 \leqq m, n \leqq k} \text { whenever each } \quad x_{m} \neq 0,
$$

* Research supported by NSERC of Canada. 
where $x=\left(x_{1}, \ldots, x_{k}\right)$ and $v_{m n}(x)=\left(x_{m}, V_{m n} x_{m}\right) . \delta_{0}$ is clearly an $H^{\times}$construction, and we label the corresponding definiteness condition $A_{0}^{ \pm}$. The corresponding $H^{\otimes}$ construction involves the determinantal operator

$$
\Delta_{0}:=\otimes \operatorname{det}\left[V_{m n}\right]_{1 \leqq m, n \leqq k}
$$

and $A_{0}^{ \pm}$is easily seen to be equivalent to definiteness of $\Delta_{0}$ on decomposable tensors of $H^{\otimes}$. The following fundamental result is due to Atkinson.

Theorem 1.1. [2; Theorem 7.8.2 and Section 8.6] $A_{0}^{ \pm}$is equivalent to definiteness of $\Delta_{0}$ on $H^{\otimes}$.

Remark. In [3] these conditions were labelled $\mathrm{RD}_{\delta}$ and $\mathrm{RD}_{\Delta}$, respectively. The notation here is chosen for unification with other conditions. Also, since we wish to discuss the logical status of Theorem 1.1, we shall retain the distinction between the two forms of the condition.

Major results of the $H^{\otimes}$ and $H^{\times}$theories under RD are as follows.

Theorem 1.2. [2; Theorem 7.6.2] If $\Delta_{0}$ is definite on $H^{\otimes}$ then for each $T=\left(T_{1}, \ldots, T_{k}\right)$ the following property of (1.1) obtains:

$\left(a_{0}^{ \pm}\right)$there exists a complete orthonormal basis of $H_{0}^{\otimes}$ consisting of decomposable tensors $x^{\otimes}=x_{1} \otimes \ldots \otimes x_{k}$ where the $x_{m}$ are eigenvectors satisfying (1.1) with $\lambda_{0} \neq 0$, and $H_{0}^{\otimes}$ is $H^{\otimes}$ endowed with the inner product $[x, y]_{0}=\left(x,\left|\Delta_{0}\right| y\right)$, where $($,$) is the Hilbert$ space inner product and $\left|\Delta_{0}\right|$ is the positive square root of $\Delta_{0}^{2}$.

We remark that Atkinson's proofs (the only ones to date) of Theorem 1.1 depend on the above result.

Theorem 1.3. [6; Theorem 2] $A_{0}^{ \pm}$implies the following property of (1.1), for each $T$ :

$\left(\hat{b}_{0}\right)$ for each $i=\left(i_{1}, \ldots, i_{k}\right)$ where the $i_{m}$ are nonnegative integers, there exists an eigenvalue $\lambda=\lambda^{i}$ satisfying (1.1) with $\lambda_{0} \neq 0$ and

$$
\rho_{m}^{i_{m}}(\lambda)=0, \quad m=1 \ldots k
$$

where

$$
\rho_{m}^{1}(\lambda) \leqq \ldots \leqq \rho_{m}^{d_{m}}(\lambda)
$$

denote the eigenvalues of $W_{m}(\lambda)$, repeated according to multiplicity.

We shall say that any $\lambda$ satisfying (1.1) and (1.4) has index $i$.

In Section 2, we shall show, roughly, that $\hat{b}_{0}$ is central to the RD theory, $\hat{b}_{0}$ for all $\boldsymbol{T}$ being equivalent to $A_{0}^{ \pm}$. We shall also show that $\bar{b}_{0}$ can be replaced by a "stable" version $b_{0+}^{s}$ where for each $i$, the $\lambda^{i}$, normalised to $\lambda_{0}^{i}=1$, are unique and continuously dependent on the $T_{m}$ and $V_{m n}$. As a consequence we shall deduce $a_{0}^{ \pm}$from $A_{0}^{ \pm}$using a minimum of tensor product machinery. In doing so, we shall reprove Theorem 1.1 via $b_{0+}^{s}$, thereby shortening the existing proofs. 
As regards equivalence with $A_{0}^{ \pm}$, we shall use the following result, which follows directly from [4; Lemma 2.3(i) and Corollary 4.4].

Theorem 1.4. $A_{0}^{ \pm}$is equivalent to the following condition on the $V_{m n}$ :

$\left(c_{0}\right) \mathbf{0} \notin R_{\sigma}$ for each $\sigma=\left(\sigma_{1}, \ldots, \sigma_{k}\right) \in \mathbb{R}^{k}$ such that $\left|\sigma_{m}\right|=1, m=1 \ldots k$.

Here $R_{\sigma}$, which was labelled $V_{\sigma}$ in [4], is defined by

$$
R_{\sigma}=\operatorname{co} \bigcup_{m=1}^{k} \sigma_{m} R_{m}, \quad R_{m}=\operatorname{co}\left\{v_{m}\left(x_{m}\right): 0 \neq x_{m} \in H_{m}\right\}
$$

where co denotes convex hull and

$$
\boldsymbol{v}_{m}\left(x_{m}\right)=\left(v_{m 1}\left(x_{m}\right), \ldots, v_{m k}\left(x_{m}\right)\right) \in \mathbb{R}^{k}
$$

We shall establish the converse of Theorem 1.3 by identifying $T(\sigma)$ and $i(\sigma)$ such that no eigenvalue of index $i(\sigma)$ exists if $0 \in R_{\sigma}$.

The remaining sections are devoted to further definiteness conditions which, in general, involve both $T$ and the $V_{m n}$. Atkinson [2; Chapters 7-10] has analysed (1.1) under three such conditions, the second and third being equivalent, essentially because of Theorem 1.1 [2; Theorem 7.8.2]. Consequences of the first condition [2; p. 117], which we label $A$, will be studied in Section 3 . We shall use analogues of Theorems 1.1 and 1.2 to prove an analogue of Theorem 1.3. As far as the author is aware, there are no previous indicial results under condition $A$. The key changes from $\hat{b}_{0}$ are (i) $\lambda_{0}$ may vanish, although some eigenvalue-independent linear combination $\mu \lambda$ will not do so (ii) $\delta_{0}(x)$ may vanish, and even if $\mu \lambda=\lambda_{0}$ (in which case we denote $A$ by $A_{0}$ ) then $\delta_{0}(x)$ may still be indefinite. We can circumnavigate (i) by a preliminary rotation of eigenvalue axes in $\mathbb{R}^{k+1}$ so as to ensure $A_{0}$, at the cost of destroying the special status of $T$. As regards (ii), we may attach signs to the eigenvalues either by $\operatorname{sgn} \delta_{0}(x)$ as in [5] or by $\sigma=\operatorname{sgn}\left(\lambda_{0} \delta_{0}(x)\right)$, assuming $A_{0}$. These two methods differ in general, and it turns out that $\sigma$ has certain advantages. In particular, $A_{0}$ implies

$\left(b_{0}\right)$ for each signed index $(i, \sigma)$, an eigenvalue $\lambda=\lambda^{(i, \sigma)}$ exists with $\lambda_{0} \neq 0$.

This will be shown in Corollary 3.7, where we shall also deduce uniqueness and continuous dependence on the data $T_{m}$ and $V_{m n}$ of each eigenvalue $\lambda=\lambda^{(i, \sigma)}$, normalised to $\left|\lambda_{0}\right|=1$.

In Section 4, we prove equivalence of $A_{0}, b_{0}$ and a "stable" version $\hat{b}_{0}^{s}$ of $\hat{b}_{0}$. We also give indicial equivalents for $A$, and for Atkinson's second condition $A^{+}$where change (i) above occurs, but (ii) does not. It turns out that one can retain the special status of $T$ at the indicial level, and that the difference between $A$ and $A^{+}$reduces to the difference between the methods described above for attaching signs to the eigenvalues. One consequence of this analysis is a new set of equivalents for $\mathrm{RD}$, in some ways more useful than those of Section 2. Finally we discuss left definiteness which is a combination of conditions on the cofactors of $\delta_{0}$ and "definiteness" of $T[3 ;$ p. 321], [8; pp. 62-3]. We shall relate the cofactor condition to $A^{+}$for all "definite" $T$, and then deduce indicial equivalents from the theory for $A^{+}$. 
Remark. Equivalents of weaker conditions on the $R_{\sigma}$ (1.5), involving the "fundamental" index $i=(1, \ldots, 1)$ for all $T$ satisfying various definiteness conditions, can be found for $k=2$ in [7], and they remain valid in infinite dimensions.

\section{Right definiteness}

In the first part of this section, we discuss some indicial equivalents for RD, beginning with a sufficiency property.

Lemma 2.1. If $\mathbf{0} \in \mathbf{R}_{\sigma}$ then there are $T=\boldsymbol{T}(\boldsymbol{\sigma})$ and $i=i(\sigma)$ so that no $\lambda=\lambda^{i}$ exists as in $\hat{b}_{0}$, i.e. (1.1), $\lambda_{0} \neq 0$ and (1.4) are incompatible.

Proof. If $0 \in R_{\sigma}$ then there exist nonnegative $a_{n_{m}}$ and nonzero $x_{n_{m}} \in H_{m}$, such that

$$
\sum_{m, n_{m}=1}^{k} \alpha_{n_{m}} \sigma_{m} \mathbf{v}_{m}\left(x_{n_{m}}\right)=\mathbf{0}, \sum_{n_{m}=1}^{k} \alpha_{n_{m}}>0
$$

in the notation of (1.5). Assuming $\lambda_{0} \neq 0$ and choosing $\boldsymbol{T}=\boldsymbol{T}(\boldsymbol{\sigma})$ so that each $\lambda_{0} \sigma_{m} T_{m}$ is negative definite, we deduce

$$
\sum_{m, n_{m}} \alpha_{n_{m}}\left(x_{n_{m}}, \sigma_{m} W_{m}(\lambda) x_{n_{m}}\right)=\sum_{m, n_{m}} \alpha_{n_{m}} \sigma_{m} \lambda_{0} t_{m}\left(x_{n_{m}}\right)<0
$$

where we write

$$
t_{m}\left(x_{m}\right)=\left(x_{m}, T_{m} x_{m}\right)
$$

Now choose $i=i(\sigma)$ so that $i_{m}=1$ if $\sigma_{m}=1$ and $i_{m}=d_{m}$ (1.2) if $\sigma_{m}=-1$. If $\lambda=\lambda^{i}$ exists as in $\hat{b}_{0}$ then (1.4) forces each $\sigma_{m} W_{m}(\lambda)$ to be nonnegative definite, and this contradicts (2.1).

We shall improve Theorem 1.3 by means of the following general perturbation result, which requires no definiteness conditions.

Lemma 2.2. The set of eigenvalues $\lambda^{i}$ of index $i$ depends upper semi-continuously on the data $T_{m}$ and $V_{m n}$.

Remark. We use the Euclidean $\mathbb{C}^{k(k+1)}$ topology for the data.

Proof. The quadratic forms $t_{m}$ and $v_{m n}$ depend continuously on the data. By the minimax principle, the same goes for the eigenvalues $\rho_{m}^{j}(\lambda)(1.4)$ for each $\lambda$. Thus the $\rho_{m}^{j}$ functions have closed graphs, and the conclusion is now immediate.

We are now ready for the first indicial equivalences. We write $b_{0+}^{s}$ to mean existence, uniqueness and continuous dependence on the data of each $\lambda^{i}$, normalised to $\lambda_{0}^{i}=1$.

Theorem 2.3 The following are equivalent: (i) $A_{0}^{ \pm}$, (ii) $b_{0}$ for all $T$, (iii) $b_{0+}^{s}$ for all $T$. 
Proof. Since (iii) $\Rightarrow$ (ii) is trivial, and Lemma 2.1 proves (ii) $\Rightarrow$ (i), it suffices to establish (i) $\Rightarrow$ (iii). Evidently $A_{0}^{ \pm}$is equivalent to $\left|\delta_{0}(u)\right|$ being positively bounded below over $\left\|u_{m}\right\|=1, m=1 \ldots k$, so continuity of $\delta_{0}$ in the data implies that $A_{0}^{ \pm}$persists under small perturbations.

Let $T_{m}(\varepsilon) \rightarrow T_{m}$ as $\varepsilon \rightarrow 0, \varepsilon \in \mathbb{R}$, and similarly for $V_{m n}(\varepsilon)$. Now the proof of Theorem 1.3 actually gives uniqueness of eigenvalues of fixed index, normalised to $\lambda_{0}=1$. By persistence of $A_{0}^{ \pm}$, there exist $\lambda^{i}(\varepsilon)$ for all suitably small $\varepsilon$, with $\lambda_{0}^{i}(\varepsilon)=1$. Let $\lambda^{i}$ be any limit point of $\lambda^{i}(\varepsilon)$ as $\varepsilon \rightarrow 0$-necessarily $\lambda_{0}^{i}=1$. Lemma 2.2 then yields uniqueness of the limit point $\lambda^{i}$, whence continuity of $\lambda^{i}(\varepsilon)$ at $\varepsilon=0$.

The remainder of this section is devoted to consequences for $H^{\otimes}$ of the indicial theory. The first step is to show that $b_{0}^{s}$ implies an $H^{\times}$version of $a_{0}^{ \pm}$.

Theorem 2.4. If $b_{0+}^{s}$ holds then there exist $x_{m}=x_{m}^{i}$ satisfying (1.1) with $\lambda=\lambda^{i}, \lambda_{0}=1$, and such that det $V^{i j}=0$ if $i \neq j$, where $V^{i j}$ is the $k \times k$ matrix with $(m, n)$ th entry $\left(x_{m}^{i}, V_{m n} x_{m}^{j}\right)$.

Proof. If $\lambda=\lambda^{i}$ corresponds to $x_{m}^{i}$ then (1.1) yields

$$
\left(W_{m}\left(\lambda^{i}\right) x_{m}^{i}, x_{m}^{j}\right)=0=\left(x_{m}^{i}, W_{m}\left(\lambda^{j}\right) x_{m}^{j}\right)
$$

whence

$$
V^{i j}\left(\lambda^{i}-\lambda^{j}\right)=0
$$

so the conclusion is immediate if the $\lambda^{i}$ are all distinct.

In the case of multiple eigenvalues, we follow Atkinson [2; Section 7.5] and perturb $T_{m}$ to $T_{m}(\varepsilon)$ so that (i) $T_{m}(\varepsilon) \rightarrow T_{m}$ as $\varepsilon \rightarrow 0$ and (ii) all the eigenvalues of (1.1) are distinct if $T_{m}$ is replaced by $T_{m}(\varepsilon)$. With $x_{m}^{i}(\varepsilon)$ as corresponding unit eigenvectors, we choose any limit points as $\varepsilon \rightarrow 0$ for the $x_{m}^{i}$. By $b_{0+}^{s}$, the $x_{m}^{i}$ do satisfy (1.1) with $\lambda=\lambda^{i}$. From the perturbed version of (2.2) we have $\operatorname{det} V^{i j}(\varepsilon)=0$ in an obvious notation, and we now let $\varepsilon \rightarrow 0$.

Remark. Although Atkinson's arguments depend explicitly on definiteness of $\Delta_{0}$ on $H^{\otimes}$, an inspection, together with the above use of indexed eigenvalues, shows that $b_{0+}^{s}$ in fact suffices.

We are now ready to draw the principal conclusions for $H^{\times}$. The following simple observation will be used at various points in the sequel.

Remark 2.5. The number of indices $i$ equals the dimension of $H^{\otimes}$.

Corollary 2.6. Theorem 1.1 holds.

Proof. Setting $x^{i \otimes}=x_{1}^{i} \otimes \ldots \otimes x_{k}^{i}$, we deduce

$$
\left(x^{i \otimes}, \Delta_{0} x^{j \otimes}\right)=0 \quad \text { if } \quad i \neq j
$$


from Theorems 2.3 and 2.4. The result now follows immediately from Remark 2.5 and the expansion

$$
\left(\sum_{i} \alpha^{i} x^{i \otimes}, \Delta_{0} \sum_{j} \alpha^{j} x^{i \otimes}\right)=\sum_{i} \delta_{0}\left(x^{i}\right)\left|\alpha^{i}\right|^{2}
$$

-see [2; Lemma 7.8.1].

Corollary 2.7. $A_{0}^{ \pm}$implies $a_{0}^{ \pm}$.

Proof. By the previous result, we may assume definiteness of $\Delta_{0}$, so $H_{0}^{\otimes}$ is constructible. Since the $x^{i \otimes}$ may be scaled to unit norm in $H_{0}^{\otimes}$ by $\left|\delta_{0}(x)\right|=1$, the result follows from (2.3) and Remark 2.5.

Remark. By utilising only [2; Lemma 7.8.1] for Corollary 2.6, we have shortened the proof of [2; Theorem 7.8.2]. Similarly the proof of Corollary 2.7 is more direct than that of [2; Theorem 7.6.2], and can be viewed as a generalisation of the procedure outlined on $[2 ;$ p. 135] for a special case.

\section{Some consequences of Condition $\boldsymbol{A}$}

Atkinson's "Definiteness Condition I" [2; p. 117], which we label $A$ here, is defined as rank $W(x)=k$ whenever each $x_{m} \neq 0$, where $W(x)$ is the $k \times(k+1)$ matrix with $m$ th row

$$
\boldsymbol{w}_{m}\left(x_{m}\right)=\left[t_{m}\left(x_{m}\right) v_{m 1}\left(x_{m}\right) \ldots v_{m k}\left(x_{m}\right)\right] .
$$

Closely associated with this condition is the determinant

$$
\delta(\boldsymbol{\mu}, x)=\operatorname{det}\left[\begin{array}{c}
\boldsymbol{\mu} \\
W(x)
\end{array}\right]
$$

where $\mu=\left[\mu_{0} \ldots \mu_{k}\right] \in \mathbb{R}^{k+1}$. The analogous $H^{\otimes}$ construction is

$$
\Delta(\mu)=\otimes \operatorname{det}\left[\begin{array}{c}
\mu \\
W
\end{array}\right]
$$

where $W$ has $m$ th row $\left[T_{m} V_{m 1} \ldots V_{m k}\right]$. Evidently

$$
\delta(\mu, x)=\left(x^{\otimes}, \Delta(\mu) x^{\otimes}\right)
$$

and it is easily seen that $A$ forces positivity of this expression for each nonzero $x_{m}$, for some $\boldsymbol{\mu}$ which in general depends on the $x_{m}$.

We start with a similar result from the $H^{\otimes}$ theory, but where $\mu$ is fixed.

Theorem 3.1. [2; Theorem 10.4.1] A implies nonsingularity of $\Delta(\mu)$ for some $\mu$. 
To make a closer parallel with Theorem 1.1, we use $e_{0}, \ldots, e_{k}$ to denote the coordinate basis of $\mathbb{R}^{k+1}$, and we write $\Delta_{n}=\Delta\left(e_{n}\right)$, in agreement with (1.3). Condition $A_{0}$ will mean $A$ and nonsingularity of $\Delta_{0}$.

Corollary 3.2. $A$ implies $A_{0}$ after a nonsingular linear eigenvalue transformation.

Indeed it suffices to rotate axes in $\mathbb{R}^{k+1}$ so that $\boldsymbol{\mu}$ in Theorem 3.1 becomes $\boldsymbol{e}_{0}$.

Two remarks are pertinent here. First, definiteness of $\Delta_{0}$ (or $\Delta(\mu)$ in Theorem 3.1) cannot be guaranteed, at least when $k>2$ [2; Section 9.9]. Second, the transformation of Corollary 3.2 may destroy the special status of $\boldsymbol{T}$. We shall return to these points in Section 4.

Our present purposes will be served by the following analogue of Theorem 1.2 , where $a_{0}$ means $a_{0}^{ \pm}$except that the inner product $(x, y)_{0}=\left(x, \Delta_{0} y\right)$ may be indefinite, so the eigenvector basis is only $H_{0}^{\otimes}$-orthogonal and not normalised to $\left|\left(x^{\otimes}, x^{\otimes}\right)_{0}\right|=1$. (Actually such normalisation is possible under $A_{0}$, as will be seen later.) We write $a$ if the eigenvalues satisfy $\mu \lambda \neq 0$ where $\mu$ is eigenvalue-independent and the (perhaps indefinite) inner product in $H^{\otimes}$ is induced by $\Delta(\mu)$ instead of $\Delta_{0}$.

Theorem 3.3. $A_{0}$ implies $a_{0}$ and $A$ implies $a$.

Proof. By [2; Theorem 10.6.1], an orthogonal basis exists as required, assuming $A$, and it remains to prove that the eigenvalues $\lambda$ satisfy $\mu \lambda \neq 0$. Now [2; Theorem 6.4.2] gives

$$
\lambda_{0} \Delta_{n} x^{\otimes}=\lambda_{n} \Delta_{0} x^{\otimes}, \quad n=1 \ldots k
$$

whenever (1.1) is satisfied. Then $A_{0}$ and $\lambda \neq 0$ force $\lambda_{0} \neq 0$. This establishes the first contention, and the second follows from

$$
(\mu \lambda) \Delta_{n} x^{\otimes}=\lambda_{n} \Delta(\mu) x^{\otimes}, \quad n=0 \ldots k .
$$

For the indicial theory in $H^{\otimes}$, it turns out to be convenient to normalise $\lambda_{0}$ (or $\mu \lambda$ ) to \pm 1 , and to specify the sign via the following construction. We define

$$
\delta_{n}(x)=\delta\left(e_{n}, x\right)=\left(x^{\otimes}, \Delta_{n} x^{\otimes}\right)
$$

and we set

$$
\delta(x)=\left[\delta_{0}(x) \ldots \delta_{k}(x)\right]^{T}
$$

In particular, $\delta(\mu, x)=\mu \delta(x)$.

Lemma 3.4. $A$ is equivalent to $\delta(x) \neq 0$ whenever each $x_{m} \neq 0$. If in addition $\lambda \in \mathbb{R}^{k+1}$ satisfies $W(x) \lambda=0 \neq \lambda$ then $\lambda=\alpha \delta(x)$ for some $\alpha \in \mathbb{R}$, and the sign of $\alpha$ depends only on $\lambda$, i.e. not on $x$. 
Proof. The first contention follows from the definition of determinant rank. For the second, $A$ implies that the $\boldsymbol{w}_{m}\left(x_{m}\right)$ (3.1) span a $k$-dimensional subspace $S \subset \mathbb{R}^{k+1}$. Thus $\lambda \in S^{\perp}$, and it suffices to prove $\delta(x) \in S^{\perp}$. Now

$$
w_{m}(x) \delta(x)=\delta\left(w_{m}(x), x\right)=0
$$

so indeed $W(x) \boldsymbol{\delta}(x)=\mathbf{0}$.

For the final contention, we note that $W(x) \lambda=0$ if and only if

$$
\left(x_{m}, W_{m}(\lambda) x_{m}\right)=0, \quad m=1 \ldots k .
$$

The set of nonzero $x_{m}$ satisfying (3.3) for each fixed $m$ is arcwise connected [2; Theorem 2.7.1]. Suppose $\lambda=\alpha \delta(x)=\beta \delta(y) \neq 0$-say $\lambda_{n} \neq 0$. Continuity of $\delta$ along an arc joining $x$ to $y$ and the hypothesis $\alpha \beta \leqq 0$ force the contradiction $\lambda_{n}=0$. Thus $\alpha \beta>0$.

This enables us to attach the sign of $\alpha$ to any $\lambda \in \mathbb{R}^{k+1}$ satisfying the conditions of Lemma 3.4. In particular, any eigenvalue satisfies those conditions under $A$ (cf. [2; p. 174]) and we refer to $(i, \sigma)$ as the signed index of $\lambda$ if $(1.4)$ holds and $\sigma=\operatorname{sgn} \alpha$. If $A_{0}$ holds then

$$
\lambda_{0}=\alpha \delta_{0}(x)
$$

for any $x_{m}$ satisfying (1.1), so since $\lambda_{0} \neq 0$ by Theorem 3.3 , we have

$$
\sigma=\operatorname{sgn}\left(\lambda_{0} \delta_{0}(x)\right) .
$$

Similarly under $A$ the sign is given by the formula

$$
\sigma=\operatorname{sgn}[(\mu \lambda)(\mu \delta(x))]
$$

for fixed $\mu$ whose existence is guaranteed by Theorem 3.1 .

Our analogue of Theorem 1.3 for $A$ will depend on the following uniqueness property.

Theorem 3.5. Assuming $A_{0}[A]$, at most one eigenvalue $\lambda$ of a given signed index, normalised to $\left|\lambda_{0}\right|=1[|\mu \lambda|=1]$ can exist.

Proof. We shall assume $A_{0}$ - the proof under $A$ is analogous. Suppose $\lambda$ and $\boldsymbol{v}$ both have signed index $(i, \sigma)$ and $\left|\lambda_{0}\right|=\left|v_{0}\right|=1$. Let $U_{m}$ denote the unit sphere of $H_{m}$ and set $U=X_{m=1}^{k} U_{m}$. Let $y_{m}$ minimise $w_{m}\left(u_{m}\right) \lambda=\left(u_{m}, W_{m}(\lambda) u_{m}\right)$ over $u_{m} \in U_{m}$ and orthogonal to the first $i_{m}-1$ eigenvectors of $W_{m}(v)$, i.e. those corresponding to $\rho_{m}^{1}(v), \ldots, \rho_{m}^{i_{m}-1}(v)$.

The minimax principle yields

$$
W(y) \lambda \leqq \mathbf{0} \leqq W(y) v
$$

coordinatewise - cf. [6; p. 1057]. Writing $\lambda^{\beta}$ for $(1-\beta) \lambda-\beta v$ we deduce

$$
W(y) \lambda^{\beta} \leqq \mathbf{0} \text { whenever } 0 \leqq \beta \leqq 1 .
$$


Similarly one may find $z \in U$ to reverse these inequalities, so

$$
W(z) \lambda^{\beta} \geqq 0 \quad \text { whenever } \quad 0 \leqq \beta \leqq 1 .
$$

Define $S(\beta)=\mathrm{X}_{m=1}^{k} S_{m}(\beta) \subset U$ by the condition

$$
u \in S(\beta) \Leftrightarrow W(u) \lambda^{\beta}=\mathbf{0} .
$$

Convexity of the numerical ranges of $W_{m}\left(\lambda^{\beta}\right), m=1 \ldots k$, together with (3.6) and (3.7), guarantee $S(\beta) \neq \emptyset$ for each $\beta \in[0,1]$. If $\lambda^{\beta}=0$ then $\mu$ and $v$ are positively proportional, and the normalisation $\left|\lambda_{0}\right|=\left|v_{0}\right|=1$ forces $\lambda=v$ as required.

To complete the proof it will therefore suffice to contradict the assumption $\lambda^{\beta} \neq \mathbf{0}$ for each $\beta \in[0,1]$. By Lemma 3.4 and (3.8), we may attach a well defined sign $\sigma(\beta)$ to each $\lambda^{\beta}$. When $\beta=0$, we use the fact that $\lambda$ has signed index $(i, \sigma)$ to deduce $\sigma(0)=\sigma$, and similarly we obtain $\sigma(1)=-\sigma$ from the sign of $v$. Define

$$
\beta^{\prime}=\inf \{\beta: \sigma(\beta)=-\sigma\} .
$$

If $\beta^{\prime}>0$, then we may choose $\beta^{j} \uparrow \beta^{\prime}$ and $u^{j} \in S\left(\beta^{j}\right)$ (3.8) so that $u_{m}^{j} \rightarrow u_{m}$ as $j \rightarrow \infty$. Evidently $\lambda^{\beta^{j}} \rightarrow \lambda^{\beta}$ and $\delta\left(u^{j}\right) \rightarrow \delta(u)$ as $j \rightarrow \infty$. Thus if $\lambda_{l}^{\beta^{\prime}} \neq 0$ then $\sigma\left(\beta^{\prime}\right)=\operatorname{sgn}\left(\lambda_{l}^{\beta^{\prime}} \delta_{0}(u)\right)=$ $\operatorname{sgn}\left(\lambda_{l}^{\beta^{j}} \delta_{0}\left(u^{j}\right)\right)=\sigma\left(\beta^{j}\right)$ for large $j$. It follows that $\sigma\left(\beta^{\prime}\right)=\sigma$, so $\beta^{\prime}=1$ contradicts $\sigma(1)=-\sigma$. If $0 \leqq \beta^{\prime}<1$ then we may use a sequence $\gamma^{j} \downarrow \beta^{\prime}$ satisfying $\sigma\left(\gamma^{j}\right)=-\sigma$ to derive the contradiction $\sigma\left(\beta^{\prime}\right)=-\sigma$.

It is now straightforward to obtain our indicial consequence of $A$.

Corollary 3.6. $A_{0}[A]$ implies the existence, uniqueness and continuous dependence on the data $T_{m}$ and $V_{m n}$ of an eigenvalue $\lambda$ for any signed index, normalised to $\left|\lambda_{0}\right|=1[|\mu \lambda|=1$ for fixed $\mu$ ].

Proof. By Theorem 3.3 there are $\operatorname{dim} H^{\otimes}$ eigenvalues, repeated according to multiplicity. Again we shall assume $A_{0}$, so each eigenvalue $\lambda$ may be normalised to $\lambda_{0}=1$ in the cited result, and each possesses a signed index by virtue of Lemma 3.4. Moreover the transformation $\lambda \rightarrow-\lambda$ changes the signed index $(i, \sigma)$ to $(d+1-i,-\sigma)$, where the $d_{m}$ satisfy $(1.2)$ and $\mathbf{1}=(1,1, \ldots, 1)$. Thus $2 \operatorname{dim} H^{\otimes}$ signed indices can be derived this way from eigenvalues, and by Remark 2.5 there are precisely $2 \operatorname{dim} H^{\otimes}$ signed indices. Existence and uniqueness now follow from Theorem 3.5.

Next we note from Lemma 3.4 that $A$ is equivalent to $\|\delta(u)\|$ being positively bounded below on $\left\|u_{m}\right\|=1, m=1 \ldots k$. This condition obviously persists under small perturbations of the data, and the proof of continuous dependence of eigenvalues now follows essentially as for Theorem 2.3 .

\section{Indicial equivalents}

It turns out that the implication of Corollary 3.6 is reversible. Moreover the full force of existence, uniqueness and continuous dependence of the $\lambda^{(i, a)}$ in not needed, and we shall explore some of the possibilities in the first part of this section. Let us begin by 
showing that existence alone, of eigenvalues $\lambda$ of all possible indices $i$ (Theorem $1.3 \mathrm{ff}$.) and admitting all possible signs of $\lambda_{0} \delta_{0}$, is not enough for $A$.

Example 4.1. Let $k=1, \quad H_{1}=\mathbb{C}^{2}, \quad T_{1}=0$ and $V_{11}\left(x^{1}, x^{2}\right)=\left(x^{1},-x^{2}\right)$ for $x=$ $\left(x^{1}, x^{2}\right) \in \mathbb{C}^{2}$.

Evidently $w_{1}(1,1)=[00]$ so $A$ fails. On the other hand let $\lambda=[10]^{T}$. Then $W_{1}(\lambda)=0$ so $\lambda$ is an eigenvalue for (1.1) with eigenspace $\mathbb{C}^{2}$. Moreover $\delta_{0}=v_{11}$ so $\delta_{0}(1,0)>0>\delta_{0}(0,1)$ and therefore all four possible combinations of index and $\operatorname{sgn} \lambda_{0} \delta_{0}$ are attained by $\lambda$. Note that $\lambda$ does not have a sign in the sense of (3.4).

It is time to detail some of the indicial conditions we shall need. By $\hat{b}$ we mean existence, for each $i$, of an eigenvalue $\lambda=\lambda^{i}$ of index $i$. If $\lambda_{0} \neq 0$ can be ensured (for some $\lambda$ for each $i$ ) then as before we write $\hat{b}_{0}$. By Example $4.1, \hat{b}_{0}$ does not imply $A$, although $\hat{b}_{0}$ for all $\boldsymbol{T}$ implies $A_{0}^{ \pm}$by Theorem 2.3 .

Two strenthenings of $\hat{b}$ that do imply $A$ are now considered.

Firstly, we assume that there exists $\mu \in \mathbb{R}^{k+1}$ such that $\mu \delta(x)$ is non-zero for all eigenvectors $x_{m}$ satisfying (1.1). Then, for the eigenvalue $\lambda=\lambda^{i}$, we can define a sign by (3.5). Indeed, elementary manipulation yields

$$
\lambda_{m}^{i} \delta_{n}(x)=\lambda_{n}^{i} \delta_{m}(x)
$$

-cf. [2, (6.8.7)]. Since $\mu \delta(x) \neq 0$ and $\lambda^{i} \neq 0$ it easily follows that $\mu \lambda^{i} \neq 0$. We write $b$ if, for each $i$, at least one such signed eigenvalue $\lambda^{i}$ exists. The sign may in principle depend on $\lambda^{i}$, although in practice Corollary 3.6 limits the possibilities.

Secondly, we write $b^{s}$ if the set of eigenvalues $\lambda^{i}$ of index $i$ is nonempty and depends continuously on the data $T_{m}$ and $V_{m n}$. By Lemma 2.2, this is equivalent to lower semicontinuity, i.e. if (1.1) is embedded in a perturbation family then each $\lambda^{i}$ is a limit point of perturbed eigenvalues of index $i$. The various strenthenings of $b$ may be applied simultaneously-e.g. $b_{0}$ means that signed eigenvalues $\lambda^{i}$ exist satisfying $\lambda_{0}^{i} \neq 0$, and $b^{s}$ means that the set of such eigenvalues $\lambda^{i}$ as in $b$ is nonempty and depends continuously on the data.

Our central result for $A$ is as follows.

Theorem 4.2. $A, b, \hat{b}^{s}$ and $b^{s}$ are equivalent.

Proof. By Corollary 3.6 and the trivial implications $b^{s} \Rightarrow b$ and $b^{s} \Rightarrow \hat{b}^{s}$, we need prove only (i) $\hat{b}^{s} \Rightarrow A$ and (ii) $b \Rightarrow A$.

Ad (i): we use the analogue of Theorem 1.4 for $A$. By [2; Theorem 9.8.1], if $A$ fails then there exist $\sigma \in \mathbb{R}^{k}$ with $\left|\sigma_{m}\right|=1$, nonnegative $\alpha_{n_{m}}$ and nonzero $x_{n_{m}} \in H_{m}$ such that

$$
\sum_{m, n_{m}=1}^{k} \alpha_{n_{m}} \sigma_{m} w_{m}\left(x_{n_{m}}\right)=0, \quad \sum_{n_{m}=1}^{k} \alpha_{n_{m}}>0
$$

in the notation of (3.1). With $i=i(\sigma)$ as in the proof of Lemma 2.1, let us suppose that an eigenvalue $\lambda$ exists with index $i$.

We claim that perturbations $W_{m}^{\varepsilon}$ of the $W_{m}$ exist such that at least one of the 
$\sigma_{m} W_{m}^{\varepsilon}(v)$ fails to be nonnegative definite for all $v$ satisfying

$$
v^{T} \lambda>0 \text {. }
$$

Note that (4.2) forces $v$ to belong to an $\varepsilon$-independent neighbourhood of $\lambda$.

Let

$$
T_{m}^{\varepsilon}=T_{m}-\varepsilon \sigma_{m} \lambda_{0} I_{m}, \quad V_{m n}^{\varepsilon}=V_{m n}-\varepsilon \sigma_{m} \lambda_{n} I_{m}
$$

where $I_{m}$ is the identity on $H_{m}$, and set

$$
W_{m}^{\varepsilon}=\lambda_{0} T_{m}^{\varepsilon}-\sum_{n=1}^{k} \lambda_{n} V_{m n}^{\varepsilon}
$$

Then we have

$$
\boldsymbol{w}_{m}^{e}\left(x_{m}\right)=\boldsymbol{w}_{m}\left(x_{m}\right)-\varepsilon \sigma_{m} \lambda\left\|x_{m}\right\|^{2}
$$

so

$$
\begin{gathered}
\sum_{m, n_{m}} \alpha_{n_{m}} \sigma_{m}\left(x_{n_{m}}, W_{m}^{\varepsilon}(v) x_{n_{m}}\right) \\
=-\varepsilon \sum_{m, n_{m}} \alpha_{n_{m}} v^{T} \lambda\left\|x_{n_{m}}\right\|^{2}
\end{gathered}
$$

by (4.1). For $\varepsilon>0$ and any $v$ satisfying (4.2), this expression is negative. Thus a neighbourhood of $\lambda$ exists containing no eigenvalue $v$ of index $i$, and therefore $\hat{b}^{s}$ must fail.

Ad (ii): again let an eigenvalue $\lambda$ exist with index $i(\sigma)$. By scaling and an axis rotation, if necessary, we may assume $\lambda=e_{0}$, so $\sigma_{m} W_{m}(\lambda)=\sigma_{m} T_{m}$ has minimal eigenvalue zero. Let $R_{\sigma}^{*}$ be defined as for $R_{\sigma}(1.5)$ but for nonzero $x_{m} \in \mathcal{N}\left(T_{m}\right)$. The condition that $\lambda$ possesses a sign forces $\delta_{0}$ not to vanish on $\mathrm{X}_{m=1}^{k} \mathcal{N}\left(T_{m}\right)$ by (3.5), and by Theorem 1.4 this implies $\mathbf{0} \notin R_{\sigma}^{*}$

On the other hand, suppose that $A$ fails. It is convenient now to restrict the index set of $n_{m}$ in (4.1) so that each $\alpha_{n_{m}}$ is positive. If

$$
\alpha_{n_{m}} \sigma_{m} w_{m}\left(x_{n_{m}}\right) \lambda=\alpha_{n_{m}} \sigma_{m} t_{m}\left(x_{n_{m}}\right)
$$

is positive for some $m$ and $n_{m}$ in (4.1) then the sum of the other terms in (4.1) is negative. This contradicts nonnegativity of the $\sigma_{m} t_{m}$, so it follows that each term $t_{m}\left(x_{n_{m}}\right)$ vanishes in (4.1). Thus each $x_{n_{m}} \in \mathcal{N}\left(T_{m}\right)$, and so (4.1) forces the contradiction $0 \in R_{\sigma}^{*}$.

Corollary 4.3. $A_{0}, b_{0}, \hat{b}_{0}^{s}$ and $b_{0}^{s}$ are equivalent.

Proof. By Corollary 3.6 and the previous result, it is enough to assume $A$ and either $b_{0}$ or $\hat{b}_{0}^{s}$, and then to deduce $A_{0}$. As in the proof of Corollary 3.6, $A$ yields precisely $2 \operatorname{dim} H^{\otimes}$ eigenvalues $\lambda$, normalised to $|\mu \lambda|=1$, with $\lambda$ as in Theorem 3.1. Thus either $\hat{b}_{0}^{s}$ or $b_{0}$ forces each eigenvalue $\mu$ to satisfy $\lambda_{0} \neq 0$. 
By [2; Theorem 6.7.2], the operators

$$
\Gamma_{n}=\Delta(\mu)^{-1} \Delta_{n}, \quad n=0 \ldots k
$$

commute. In particular, $\mathscr{N}\left(\Gamma_{0}\right)=\mathscr{N}\left(\Delta_{0}\right)$ is invariant for each $\Gamma_{n}$. If $\mathscr{N}\left(\Gamma_{0}\right)$ is nontrivial then the $\Gamma_{n}$ have a common eigenvector with eigenvalues $v_{n}$, say, where $v_{0}=0$. By [2; Theorem 6.8.1], $v$ is an eigenvalue for (1.1) so $v_{0}=0$ gives a contradiction. It follows that $\mathscr{N}\left(\Delta_{0}\right)$ must be trivial.

We turn now to Atkinson's "Definiteness Condition II" [2; p. 121] which we label $A^{+}$ here. This requires the existence of $\mu \in \mathbb{R}^{k+1}$ such that

$$
\delta(\mu, x)>0 \text { for all nonzero } x_{m} \in H_{m}, \quad m=1 \ldots k .
$$

The corresponding analogue of Theorem 1.1 is [2; Theorem 7.8.2] that $A^{+}$implies positive definiteness of $\Delta(\mu)$ on $H^{\otimes}$. When $\mu= \pm e_{0}$, we write $A_{0}^{ \pm}$for $A^{+}$, in agreement with Sections 1 and 2.

As we have seen, if $b$ holds then so does $A$, and the eigenvalues $\lambda$ may be partitioned into two subsets by the sign of either $\mu \lambda$ or $(\mu \lambda)(\mu \delta(x)), \mu$ as in Theorem $3.1, x_{m}$ as in (1.1). Our specialisation $b^{+}$of $b$ for $A^{+}$requires these methods of partitioning to coincide. In other words, $b^{+}$means for each $i$ the existence of at least one eigenvalue $\lambda$ of index $(i,+)$ such that $\mu \lambda>0, \mu$ being independent of $i$. Suffix ${ }_{0}$ continues to refer to $\mu=e_{0}$, and superfix ${ }^{s}$ to continuous dependence on the data.

Corollary 4.4. $A^{+}, b^{+}$and $b^{+s}$ are equivalent.

Proof. By an axis rotation, we may assume $\mu=e_{0}$, and by Corollary $4.3, A_{0}^{+}$implies $b_{0}$. As in the proof of Corollary 3.6, there is a (unique) eigenvalue $\lambda$ of signed index $(i,+)$. With $x_{m}$ as in $(1.1)$, we have

$$
\begin{aligned}
\operatorname{sgn} \lambda_{0} & =\operatorname{sgn}\left(\lambda_{0} \delta_{0}(x)\right) & & \text { by } A_{0}^{+} \\
& =1 & & \text { by definition. }
\end{aligned}
$$

Thus $b_{0}^{+}$holds, and continuous dependence comes from Corollary 3.6.

Conversely, $b_{0}^{+}$implies $A$ by Corollary 4.3. If $\lambda$ has index $(i,+)$ and $\lambda_{0}=1$ then

$$
\operatorname{sgn} \delta_{0}(x)=\operatorname{sgn}\left(\lambda_{0} \delta_{0}(x)\right)=1
$$

so $\delta_{0}(x)>0$. The proof of Corollary 2.6 then completes the argument.

We are now in a position to reexamine RD, and first we summarise some of its equivalents. By $b_{0}^{ \pm}$we mean $b^{+}$for $\mu= \pm e_{0}$ and $b_{0}^{ \pm s}$ is analogous.

Corollary 4.5. $A_{0}^{ \pm}$is equivalent to (i) $\hat{b}_{0}$ for all $\boldsymbol{T}$ (ii) $b$ for $\boldsymbol{T}=\mathbf{0}$ (iii) $b_{0}^{ \pm}$for any fixed $\boldsymbol{T}$ (iv) $b_{0}^{ \pm s}$ for all $T$. 
Proof. We note that $A_{0}^{ \pm}$is equivalent to $A$ when $\boldsymbol{T}=\mathbf{0}$. Thus the result follows from Theorems 2.3 and 4.2 and Corollary 4.4 .

Formally, (iv) includes the other consequences of RD, and even includes uniqueness of the normalised eigenvalues (cf. $b_{0+}^{s}$ in Theorem 2.3), by virtue of Corollary 3.6. Let us compare (i), (ii) and (iii) as sufficient conditions for RD. (i) involves checking all eigenvalues for all $T$ (actually for the $2^{k}$ choices $T(\sigma)$ in the proof of Lemma 2.1). (ii) and (iii) both involve checking two scalars for all eigenvalues (actually for $2^{k}$ indices $i(\sigma))$ for a single choice of $T$ ). These checks are significantly easier than those in (i).

Remark 4.6. The formal transcription of Corollary 4.5 to $A^{+}$involves replacing $T$ by a linear combination of the columns in $W$, so $T$ loses its special status. This loss is not explicit in Corollary 4.5 , but is implicit because $\lambda_{0}$ plays a special rôle. In particular, $\lambda_{0}=0$ changes the nature of (1.1) as far as $T$ is concerned. One can circumnavigate this by suppressing such eigenvalues, which correspond to $\mathscr{N}\left(\Delta_{0}\right)$ - see (3.2). Then we may scale $\lambda_{0}$ to unity, and write $\hat{\lambda}$ for the "inhomogeneous" eigenvalue $\left[\lambda_{1} \ldots \lambda_{k}\right]^{T}$. We may also rotate $\mu$ to say $e_{k}$ by what is now an affine transformation of $\hat{\lambda}$ space $-\mathrm{cf}$. [4; Lemma 5.1]. Then $H_{k}^{\otimes}$ may be constructed as $H^{\otimes}$ under the inner product $(x, y)_{k}=\left(x, \Delta_{k} y\right)$, and the $\Gamma_{n}$ (4.3) are Hermitian in $H_{k}^{\otimes}$. Commutativity of the $\Gamma_{n}$ implies that the eigenvectors $x^{\otimes}$ corresponding to "inhomogeneous" eigenvalues span $\mathcal{N}\left(\Gamma_{0}\right)^{\perp}=\mathcal{N}\left(\Delta_{0}\right)^{\perp}$ in $H_{k}^{\otimes}$. The sign of an eigenvalue $\hat{\lambda}$ can be obtained from the sign of $\delta_{0}(x)$, since

$$
\operatorname{sgn} \delta_{0}(x)=\operatorname{sgn}\left(\lambda_{0} \delta_{0}(x)\right)=\operatorname{sgn}\left(\lambda_{k} \delta_{k}(x)\right)
$$

Thus an indicial theory can be constructed for $\mathrm{A}^{+}$, via Corollary 4.4, preserving the special status of $\boldsymbol{T}$ (and $\lambda_{0}$ and $\delta_{0}$ ). This extends a corresponding construction for the left definite case-see [5] where a signed index was introduced for inhomogeneous eigenvalues, based on $\delta_{0}$.

We conclude with an indicial equivalent for left definiteness, which is the combination [3; p. 321], [8; pp. 62-3] of:

(i) (definiteness of $T$ ) all $T_{m}$ are nonnegative definite, with at least one positive definite.

(ii) (cofactor condition) for some $\mu \in \mathbb{R}^{k+1}$ and for each $m=1 \ldots k$, the determinant $\delta_{0}(x)$, with $m$ th row replaced by $\left[\mu_{1} \ldots \mu_{k}\right]$, is positive for all nonzero $x_{m} \in H_{m}$. It is convenient to set the arbitrary $\mu_{0}$ to zero.

Corollary 4.7. The cofactor condition is equivalent to $b^{+}$for all definite $T$, with the proviso that $\mu$ in the definition of $b^{+}$may be chosen with $\mu_{0}=0$ and independently of $T$.

Proof. A simple computation [3; equation (1.6)] shows that left definiteness implies $A^{+}$, with $\mu$ as in the cofactor condition, so $b^{+}$for all definite $T$ follows from Corollary 4.4. Conversely, $b^{+}$for all definite $T$ implies $A^{+}$for all definite $T$ by the same result, with $\mu$ as in the definition of $b^{+}$.

We claim that the cofactor condition holds for the same $\mu$. Indeed if not then

$$
\sum_{n=1}^{k} \mu_{n} \delta_{0 l n}(x) \leqq 0
$$


for some $l$ and some nonzero $x_{m} \in H_{m}$, where $\delta_{01 n}(x)$ is the $(l, n)$ cofactor of $\delta_{0}(x)$. Choose $T_{l}$ positive definite, with the other $T_{m}=0$. Then

$$
\begin{aligned}
\delta(\mu, x) & =\mu_{0} \delta_{0}(x)+\sum_{m, n=1}^{k} t_{m}(x) \mu_{n} \delta_{0 m n}(x) \\
& =t_{l}(x) \sum_{n=1}^{k} \mu_{n} \delta_{0 l n}(x) \leqq 0
\end{aligned}
$$

and this contradicts $A^{+}$for the given choice of $\mu$.

Remark. Further equivalences involving uniqueness and continuous dependence of the eigenvalues of index $(i,+)$ follow from Corollaries 3.6 and 4.4.

Acknowledgement. Preliminary work on right and left definiteness for the case $k=2$ was carried out in collaboration with Dr. L. Turyn, via the method of eigencurve intersections.

\section{REFERENCES}

1. F. V. Atkinson, Discrete and Continuous Boundary Problems (Academic Press, 1964).

2. F. V. AtKinson, Multiparameter Eigenvalue Problems vol. 1 (Academic Press, 1972).

3. P. A. Binding Multiparameter definiteness conditions, Proc. Roy. Soc. Edin. 89A (1981), 319332.

4. P. A. Binding, Multiparameter definiteness conditions II, Proc. Roy. Soc. Edin. 93A (1982), 47-61.

5. P. A. Binding, Abstract oscillation theorems for multiparameter eigenvalue problems, J. Diff. Equations 49 (1983), 331-343.

6. P. A. Binding and P. J. BRowne, A variational approach to multiparameter eigenvalue problems in Hilbert space, SIAM J. Math. Anal. 9 (1978), 1054-1067.

7. P. A. Binding, P. J. Browne and L. Turyn, Existence conditions for two parameter eigenvalue problems, Proc. Roy. Soc. Edin. 91A (1981), 15-30.

8. B. D. Sleeman, Multiparameter Eigenvalue Problems in Hilbert Space (Pitman, 1978).

Department of Mathematics and Statistics

UNIVERSITY OF CALGARY 